\title{
The relationship between asymptomatic organ damage, and serum soluble tumor necrosis factor-like weak inducer of apoptosis (sTWEAK) and Interleukin-17A (IL-17A) levels in non-diabetic hypertensive patients
}

\author{
Ihsan Ates ${ }^{1 *}$, Nihal Özkayar ${ }^{1}$, Fatma Akyel ${ }^{1}$, Canan Topcuoglu² ${ }^{2}$ Serdar Akyel ${ }^{3}$, A Nurdan Barça ${ }^{4}$ and Fatih Dede ${ }^{1}$
}

\begin{abstract}
Background: This study aimed to measure the serum soluble tumor necrosis factor-like weak inducer of apoptosis (sTWEAK) and interleukin-17A (IL-17A) levels in hypertensive patients with/without asymptomatic organ damage (AOD), as well as to determine the relationship between the serum sTWEAK and IL17-A levels, and carotid intima media thickness (CIMT), proteinuria, retinopathy, and the left ventricle mass index (LVMI).

Methods: The study included 159 patients diagnosed with and followed-up for primary hypertension (HT); 79 of the patients had AOD (61 female and 18 male) and 80 did not (52 female and 28 male). sTWEAK and IL-17A levels were measured in all patients.

Results: The STWEAK level was significantly lower in the patients with AOD than in those without AOD $(858.4 \mathrm{pg} / \mathrm{mL}$ vs. $1151.58 \mathrm{pg} / \mathrm{mL}, P=0.001)$. The sTWEAK level was negatively correlated with the mean microalbuminuria level and LVMI. The median IL-17A level was significantly higher in the patients with AOD than in those without AOD (2.34 pg/ $\mathrm{mL}$ vs. $1.80 \mathrm{pg} / \mathrm{mL}, \mathrm{P}=0.001$ ). There was a positive correlation between mean IL-17A level, and mean microalbuminuria level, CIMT, and LVMI. Multivariate logistic regression analysis showed that patient age, sTWEAK level, and mean 24-h systolic blood pressure were predictors of AOD.
\end{abstract}

Conclusions: The sTWEAK level was lower and IL-17A level was higher in the patients with AOD. It remains unknown if sTWEAK and IL-17A play a role in the pathophysiology of AOD. Prospective observational studies are needed to determine the precise role of sTWEAK and IL-17A in the development of target organ damage.

Keywords: Asymptomatic organ damage, Hypertension, Interleukin-17, Tumor necrosis factor-like weak inducer of apoptosis

\section{Background}

Hypertension (HT) is a major risk factor for cardiovascular events (e.g. stroke, heart attack, sudden death, heart failure, and peripheral artery disease) and end-stage renal disease [1]. In addition to accelerated atherosclerosis, HT causes organ damage, including left ventricle hypertrophy, renal dysfunction, arterial aneurysm, and retinopathy, resulting

\footnotetext{
*Correspondence: dr.ihsanates@hotmail.com

'Department of Nephrology, Ankara Numune Education and Research Hospital, Sihhiye, Ankara 061100, Turkey

Full list of author information is available at the end of the article
}

in morbidity and mortality. Although HT is a serious disease, its etiopathogenesis remains largely unknown. While historically an emphasis has been placed on the role of salt intake, obesity, insulin resistance, the renin-angiotensin system, and the sympathetic nervous system in the etiopathogenesis of HT [2], endothelial dysfunction and immune mechanisms have recently become the focus of greater attention [3]. In addition, recent studies have examined the association between HT, and T lymphocytes and cytokines [4-7]. 
Tumor necrosis factor-like weak inducer of apoptosis (TWEAK) is a member of the tumor necrosis factor (TNF) superfamily that was first described in 1997 [8]. As other members of the TNF superfamily, TWEAK has multiple forms: mTWEAK, which is bonded to the membrane, and STWEAK, which is a soluble variant produced following proteolytic cleavage by endoprotease furin. Both of these forms are biologically active [9]. sTWEAK binds to fibroblast growth factor-inducible molecule 14 (Fn14) receptors, and plays a role in cellular proliferation, migration, apoptosis, differentiation, angiogenesis, and inflammation via the nuclear factor $\mathrm{kB}$ (NFкB) pathway [10].

In 2005 interleukin-17 (IL-17) was known as a cytokine produced only by $\mathrm{T}$ helper 17 [11], but more recent studies have shown that it can be produced by macrophages, dendritic cells, natural killer cells, natural killer T cells, and $\gamma \delta \mathrm{T}$ cells. There are 6 known types of IL-17 (IL-17A-F); the prototype is IL-17A [5]. IL-17A is known to play a role in autoimmune and inflammatory diseases, including rheumatoid arthritis, psoriasis, multiple sclerosis, asthma, and inflammatory bowel disease [12,13], and in chronic vascular inflammation, atherosclerosis, and hypertensive vascular changes [14]. The literature does include studies on STWEAK and IL-17A in patients with HT [4,5,7], but does not include any studies on the relationship between sTWEAK and IL-17A, and asymptomatic organ damage (AOD) in HT patient.

As such, the present study aimed to measure serum levels of sTWEAK and of IL-17A in patients with primary HT -with/without AOD- as well as to determine the relationship between the serum STWEAK and IL17A levels, and such markers of AOD as carotid intima media thickness (CIMT), proteinuria, retinopathy, and the left ventricular mass index (LVMI).

\section{Methods}

This study was conducted at the Ankara Numune Education and Research Hospital, Nephrology Clinic, Ankara, Turkey, between July 2013 and November 2013. The study was performed in accordance with the Declaration of Helsinki and the study protocol was approved by the Ankara Numune Education and Research Hospital Ethics Committee. All the patients provided written informed consent to participate in the study.

The study included 159 patients aged $>18$ years that were previously diagnosed as HT; 79 patients with AOD (18 male and 61 female) (based on grade III-IV retinopathy, LVMI $>95 \mathrm{~g} / \mathrm{m}^{2}$ for females and $>115 \mathrm{~g} / \mathrm{m}^{2}$ for males, CIMT $>0.9 \mathrm{~mm}$ or plaque, microalbuminuria (30-300 $\mathrm{mg} /$ 24-h) and 80 patients without AOD (28 male and 52 female). Patients with secondary HT, dyslipidemia (lowdensity lipoprotein [LDL] cholesterol $>130 \mathrm{mg} / \mathrm{dL}$, triglycerides [TG] $>150 \mathrm{~g} / \mathrm{dL}$ ), diabetes mellitus, acute renal injury, nephrotic proteinuria, coronary heart disease, heart failure, peripheral artery disease, cerebrovascular events, malignancy, liver disease, rheumatic diseases, thyroid diseases, and cigarette and alcohol use were excluded.

Duration of HT (years) was calculated based on patient self-reports of the date they were first diagnosed as HT to the date of inclusion in the study. The types of antihypertensive drugs the patients used included renin angiotensin system (RAS) blockers, calcium channel blockers (CCB), beta blockers, and diuretics.

The WatchBP 03 device (Microlife WatchBP AG, Switzerland) was used for 24-h ambulatory blood pressure monitoring (ABPM). Patients were informed about the procedure and the device; they were instructed to perform their daily activities as normal, but to remain immobile while the device was recording measurements. The devices were programmed to record blood pressure measurements every $15 \mathrm{~min}$ from 07.00 to 23.00 (daytime) and every $20 \mathrm{~min}$ from 23.00 to 07.00 (nighttime). The method was considered reliable if $>70 \%$ of measurements were valid.

\section{Biochemical parameters}

Blood samples for analysis of biochemical parameters were collected in the morning following a 12-h fasting. Collected blood was immediately centrifuged at $4000 \mathrm{rpm}$ for $10 \mathrm{~min}$ to separate the plasma and serum, and serum was stored at $-80^{\circ} \mathrm{C}$ until analyzed. All biochemical parameters were analyzed in the same serum sample. Patients were asked to collect 24-h urine samples; they were instructed not to collect urine from the first urination after waking the morning of the day they started to collect the urine, to urinate into a collection container during every urination thereafter, including the first urination upon waking the following morning, and to bring all the collected urine to the laboratory.

Photometric analysis of hemoglobin was performed using a Sysmex XE 2100 (Roche Diagnostics Corp. Indiana, USA) hematology auto-analyzer, 24-h urine protein was measured via the microalbumin turbidimetric method, creatinine and total protein were measured using the albumin colorimetric method, uric acid, fasting glucose, TG and total cholesterol were measured via the enzymatic colorimetric method, and high-density lipoprotein (HDL) cholesterol was measured via the homogenous enzymatic colorimetric method using a Hitachi Modular P800 (Roche Diagnostics Corp. Indiana, USA) auto-analyzer. LDL cholesterol was calculated using the Friedewald method [15]. The GFR (glomerular filtration rate) was calculated according to the simplified version of the Modification of Diet in Renal Disease Study prediction equation formula, as follows: GFR $=186 \times$ creatinine $^{-1.154} \times$ age $^{-0.203} \times 1.212$ (if African - American) $\times$ 0.742 (if female) [16]. 


\section{sTWEAK measurement}

Serum sTWEAK $(\mathrm{pg} / \mathrm{mL})$ was measured using a serum ELISA kit (eBioscience, Human TWEAK Instant ELISA, cat. no. BMS2006INST). The calculated overall intra-assay and inter-assay coefficients of variation were $7.9-9.2 \%$.

\section{IL-17A measurement}

Serum IL-17A $(\mathrm{pg} / \mathrm{mL})$ was measured using a serum ELISA kit (eBioscience, human IL-17A platinum ELISA, cat. no. BMS2017). The calculated overall intra-assay and interassay coefficients of variation were $7.1-9.1 \%$.

\section{Fundoscopic examination}

Into both eyes of each patient, 1 drop of tropicamide 1\% solution was administrated $15 \mathrm{~min}$ prior to funduscopic examination. Funduscopic examination was performed using a biomicroscopy device (Topcon SL-3C, Topcon Corporation, Japan). Retinopathy staging was performed in accordance with ESC/ESH 2013 hypertension guidelines, as follows: grade I: focal or general arteriolar narrowing; grade II: arteriovenous nicking; grade III: retinal hemorrhages, microaneurysms, hard exudates, and cotton wool spots; grade IV: grade III signs and papilledema and/or macular edema) [1].

\section{Echocardiographic examination}

Echocardiography was performed by an investigator blinded to each patient's clinical status, using a commercial echocardiography machine (Vivid 7, GE Vinged Ultrasound AS, Horten, Norway) equipped with a $2.5-\mathrm{MHz}$ phased array transducer. Complete 2D, color, pulsed, and continuous-wave Doppler examinations were performed according to standard techniques. The LVMI was calculated from 2D echocardiographic measurements, using the Devereux formula, and was indexed to body surface area, as follows: LVMI $=1.04 \times$ [(IVST + PWT + LVDd)3 - (LVDd)3] - 13.6 [17].

\section{Carotid ultrasonography}

CIMT was measured with patients in the supine position with their hand bent backwards. One radiologist that was blinded to the clinical status of the patients used a highresolution B-mode ultrasound device (Logic 7, GE Med. Inc., USA), a linear probe on the right and left common carotid artery, and an automated system. Measurements were made at 3 points: the right and left common carotid artery, bifurcation, and first $2 \mathrm{~cm}$ of the internal carotid artery. Longitudinal measurements were made from the distances defined as vessel lumen echogenicity and media-adventitia echogenicity. Mean CIMT was calculated by averaging 3 measurements made at each carotid artery [18].

\section{Statistical analysis}

Statistical analysis were performed using Statistical Package for Social Sciences (SPSS) v.20.0 for Windows (SPSS,
Inc., Chicago, IL). Kolmogorov-Smirnov test was used to analyze data with normal distribution. Numeric parameters with normal distribution are presented as mean \pm standard deviation (SD) and numeric parameters without normal distribution are presented as median. Categorical variables are expressed as number and percentage. Correlations between numeric parameters were analyzed via Pearson's and Spearman's correlation analysis. The chisquare test and Fisher's exact chi-square test were used to compare categorical variables. Between-group differences in numeric parameters with normal distribution were analyzed using the independent samples $t$ test, and betweengroup differences in numeric parameters without normal distribution were analyzed using the Mann-Whitney $U$ test. The effect of risk factors associated with AOD alone was analyzed via univariate logistic regression analysis. Significant variables based on logistic regression analysis were incorporated into a multivariate logistic regression model. ROC (receiver operating characteristic) curve analysis was used to determine the value of the serum sTWEAK level in predicting AOD. To determine the prediction point the point with the highest sensitivity and specificity, and the lowest false positive and false negative predictive points were selected. The level of statistical significance was set at $\mathrm{P}<0.05$

\section{Results}

The study included 159 patients; 79 patients with AOD (18 male and 61 female; mean age: $54.7 \pm 12.0$ years) and 80 patients without AOD (28 male and 52 female; mean age: $49.4 \pm 11.5$ years). There weren't any significant differences in demographic data between the 2 groups, except for mean age and median duration of HT $(\mathrm{P}<0.05)$. Patients in both groups used RAS blockers, CCBs, beta blockers, and diuretics as antihypertensive drugs. Although mean 24-h systolic blood pressure (SBP) and 24-h diastolic blood pressure (DBP) were significantly higher in the AOD group, there weren't any significant differences between groups according to the type of antihypertensive drugs used $(P>0.05)$. Demographic and laboratory findings in both groups are presented in Table 1.

In evaluation of all patients for types of antihypertensive drugs used, means of 24 -h SBP and 24-h DBP for patients received RAS blocker, CCB, beta blocker and diuretics were similar $(\mathrm{P}>0.05)$.

Mean 24-h SBP and 24-h DBP were significantly higher in the AOD group (24-h SBP: $129.5 \mathrm{mmHg} \pm 16.8 \mathrm{mmHg}$ vs. $119.2 \mathrm{mmHg} \pm 9.9 \mathrm{mmHg}, \quad \mathrm{P}=0.001 ; 24-\mathrm{h} \mathrm{DBP}$ : $79.5 \mathrm{mmHg} \pm 11.8 \mathrm{mmHg}$ vs. $76.1 \mathrm{mmHg} \pm 6.9 \mathrm{mmHg}$, $\mathrm{P}=0.029$ ). Median serum sTWEAK was significantly lower in the AOD group (858.4 pg/mL vs. $1151.58 \mathrm{pg} / \mathrm{mL}$, $\mathrm{P}=0.001$ ) (Figure 1). Median IL-17A was significantly higher in the AOD group $(2.34 \mathrm{pg} / \mathrm{mL}$ vs. $1.80 \mathrm{pg} / \mathrm{mL}$, $\mathrm{P}=0.001$ ) (Figure 2). 
Table 1 Patient demographic and laboratory findings

\begin{tabular}{|c|c|c|c|c|}
\hline Variables & All patients & Patients with AOD & Patients without AOD & $\mathbf{P}$ \\
\hline $\mathrm{n}(\%)$ & 159 & $79(49.6 \%)$ & $80(50.4 \%)$ & \\
\hline Age (years) & $52.02 \pm 12.1$ & $54.71 \pm 12.0$ & $49.36 \pm 11.5$ & $0.005^{*}$ \\
\hline Gender & & & & 0.115 \\
\hline Female, n (\%) & $113(71.1)$ & $61(77.2)$ & $52(65)$ & \\
\hline Male, n (\%) & $46(28.9)$ & $18(22.8)$ & $28(35)$ & \\
\hline $\mathrm{BMI}\left(\mathrm{kg} / \mathrm{m}^{2}\right)$ & $29.55 \pm 4.7$ & $30.04 \pm 4.7$ & $29.05 \pm 4.6$ & 0.195 \\
\hline Duration of HT (years) & $3.74(4)$ & $5(3)$ & $2.5(4)$ & $0.037^{*}$ \\
\hline \multicolumn{5}{|l|}{ Antihypertensive drugs } \\
\hline \multicolumn{5}{|l|}{ Class $(n, \%)$} \\
\hline RAS blocker & $86(54.1)$ & $45(57.0)$ & $41(51.2)$ & 0.470 \\
\hline Beta blocker & $28(17.6)$ & $17(21.5)$ & $11(13.8)$ & 0.198 \\
\hline $\mathrm{CCB}$ & $63(39.6)$ & $35(44.3)$ & $28(35.0)$ & 0.230 \\
\hline Diuretic & $54(34)$ & $26(33.8)$ & $28(36.8)$ & 0.691 \\
\hline CIMT (mm) & $0.75 \pm 0.17$ & $0.80 \pm 0.2$ & $0.68 \pm 0.1$ & $0.001^{*}$ \\
\hline LVMI $\left(\mathrm{g} / \mathrm{m}^{2}\right)$ & $90.05 \pm 21.1$ & $100.57 \pm 21.1$ & $78.64 \pm 13.9$ & $0.001^{*}$ \\
\hline Microalbuminuria (mg/d) & $10(15,8)$ & $19.32(41,7)$ & $7.17(6,9)$ & $0.001^{*}$ \\
\hline Proteinuria (mg/d) & $94.98(70.4)$ & $111.25(114.8)$ & $84.25(59.1)$ & $0.001^{*}$ \\
\hline Retinopathy (n,\%) & & & & $0.002^{*}$ \\
\hline None & $91(57.2)$ & $39(49.4)$ & $52(65.0)$ & \\
\hline Grade I & $28(17.6)$ & $13(16.5)$ & $15(24.6)$ & \\
\hline Grade II & $28(17.6)$ & $15(19.0)$ & $13(21.3)$ & \\
\hline Grade III & $12(7.5)$ & $12(15.2)$ & - & \\
\hline sTWEAK (pg/mL) & $961.12(560.9)$ & $858.4(365.2)$ & $1151.58(598.4)$ & $0.001^{*}$ \\
\hline IL-17A (pg/mL) & $2.1(0,9)$ & $2.34(0,9)$ & $1.80(0,7)$ & $0.001^{*}$ \\
\hline 24-h SBP (mmHg) & $124.31 \pm 14.7$ & $129.53 \pm 16.8$ & $119.16 \pm 9.9$ & $0.001^{*}$ \\
\hline 24-h DBP (mmHg) & $77.83 \pm 9.8$ & $79.53 \pm 11.8$ & $76.15 \pm 6.9$ & $0.029^{*}$ \\
\hline
\end{tabular}

Numeric parameters are shown as mean \pm SD and median (interquartile range). Categorical parameters are shown as $\mathrm{n}(\%)$.

*Denotes a significant difference between the groups $(P<0.05)$.

The serum sTWEAK level was negatively correlated with the microalbuminuria level, proteinuria level, LVMI, mean 24-h SBP, and duration of HT $(\mathrm{P}<0.05)$; however, the serum STWEAK level was not correlated with CIMT, retinopathy stage, or mean 24-h DBP $(P>0.05)$. There was a weak negative correlation between the serum sTWEAK level and patient age $(\mathrm{r}=-0.183, \mathrm{P}=0.021)$.

There was a positive correlation between the IL-17A level, and the microalbuminuria level, proteinuria level, CIMT, LVMI, and mean 24-h SBP $(\mathrm{P}<0.05)$; however, retinopathy stage and mean $24-\mathrm{h}$ DPB were no correlated with the IL-17A level $(P>0.05)$. There was a weak positive correlation between the IL-17A level and patient age $(r=0.184, P=0.020$, and a moderately negative correlation between the serum sTWEAK level and IL-17A level $(\mathrm{r}=-0.354, \mathrm{P}<0.05)$.

Mean age of HT patients with AOD was significantly higher than of those without AOD $(54.71 \pm 12.0$ vs
$49.36 \pm 11.5 ; \mathrm{p}=0.005)$. The age factor was also included in the regression model and thus it is determined that decreased sTWEAK level independent of age increased the risk for AOD $(\mathrm{OR}=0.985, \mathrm{p}=0.002)$.

Mean 24-h SBP was positively correlated with the microalbuminuria level, proteinuria level, CIMT, and LVMI $(\mathrm{P}<0.05)$; however, retinopathy stage and mean 24-h SBP were not correlated $(\mathrm{P}>0.05)$. In addition, there wasn't a correlation between mean 24-h SBP and patient age $(\mathrm{r}=-0.027, \mathrm{P}=0.733)$. There was a positive correlation between mean 24-h DBP, and the microalbuminuria level and CIMT $(\mathrm{P}<0.05)$; however, retinopathy stage was not correlated with proteinuria or LVMI ( $\mathrm{P}>$ $0.05)$. Table 2 shows the correlation analyses in detail. Retinopathy stage $\geq$ III, microalbuminuria, proteinuria, LVMI, and CIMT findings were not included in the logistic regression analysis, as they were used to identify target organ damage. 


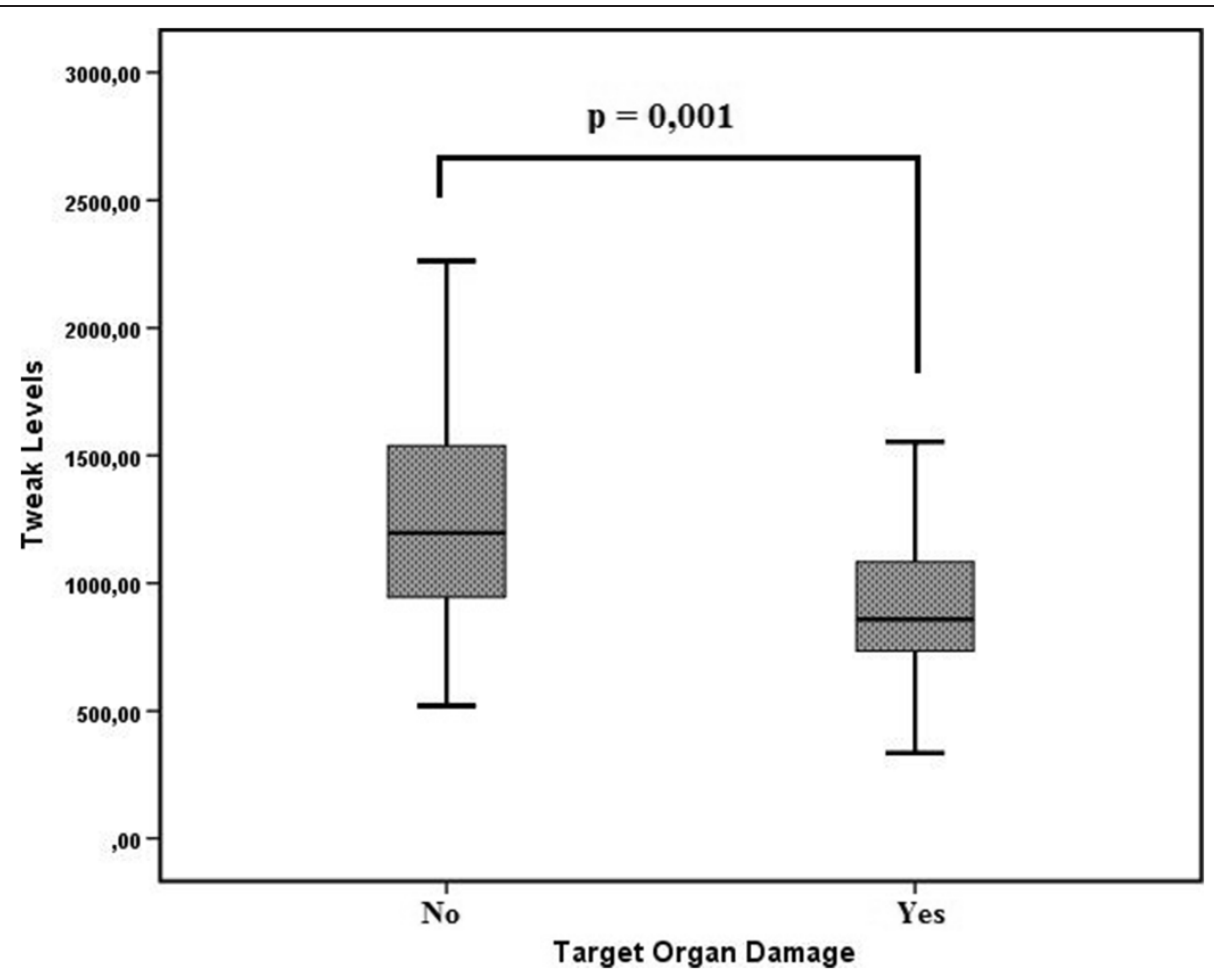

Figure 1 Between-group comparison of the serum sTWEAK level.

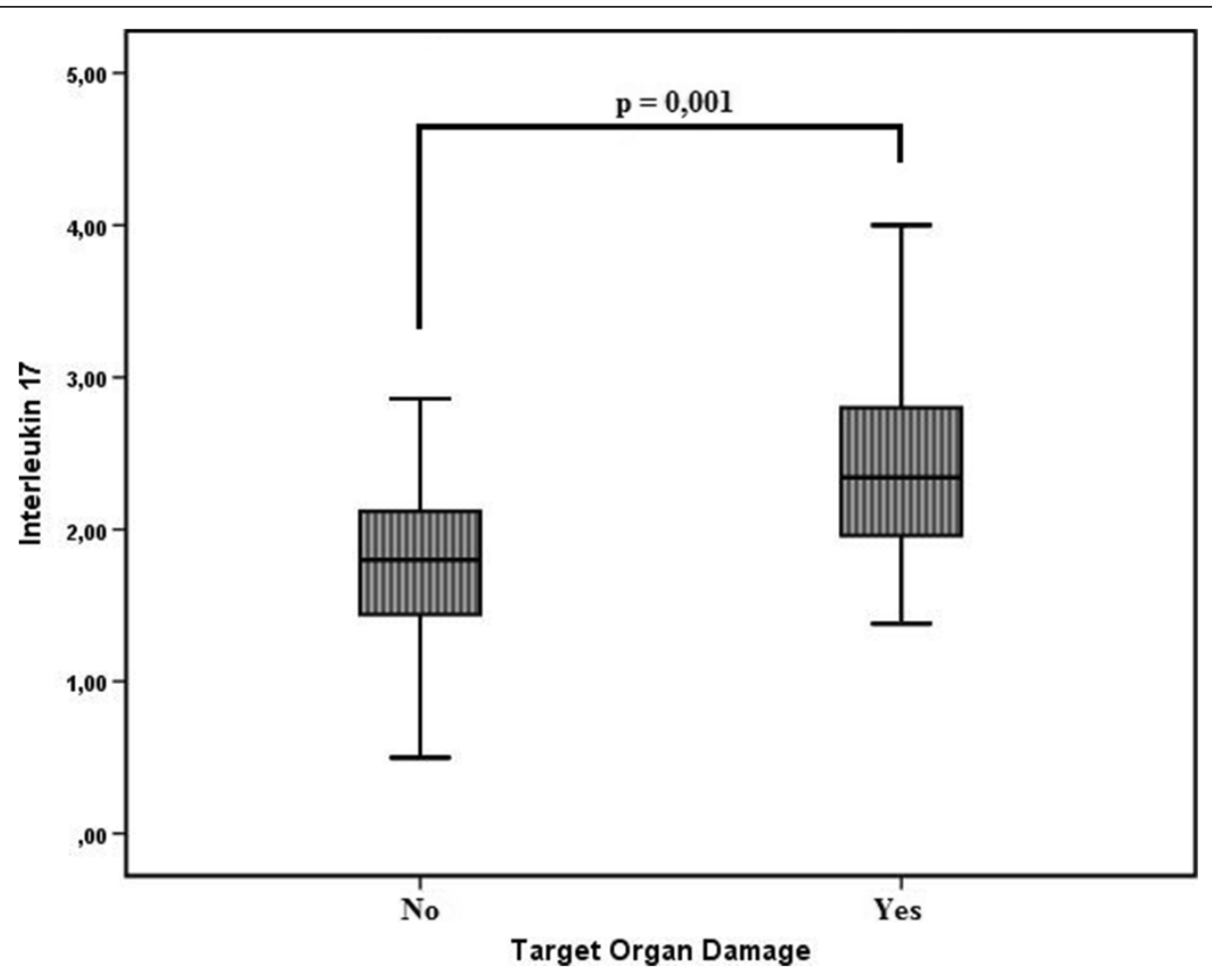

Figure 2 Between-group comparison of the serum IL-17A level. 
Table 2 Correlation analysis of sTWEAK, IL-17A, 24-h SBP, and 24-h DBP, and the risk factors

\begin{tabular}{|c|c|c|c|c|c|c|c|c|}
\hline \multirow[t]{2}{*}{ Variables } & \multicolumn{2}{|l|}{ sTweak } & \multicolumn{2}{|l|}{ IL17-A } & \multicolumn{2}{|c|}{ 24-h SBP } & \multicolumn{2}{|c|}{ 24-h DBP } \\
\hline & $r$ & $\mathbf{P}$ & $r$ & $\mathbf{P}$ & $\mathbf{r}$ & $\mathbf{P}$ & $r$ & $\mathbf{P}$ \\
\hline Microalbuminuria & -0.188 & $0.024^{*}$ & 0.253 & $0.002^{*}$ & 0.430 & $0.001^{*}$ & 0.241 & $0.004^{*}$ \\
\hline Proteinuria & -0.165 & $0.048^{*}$ & 0.193 & $0.023^{*}$ & 0.195 & $0.019^{*}$ & 0.144 & 0.084 \\
\hline CIMT & -0.104 & 0.340 & 0.229 & $0.036^{*}$ & 0.366 & $0.001^{*}$ & 0.217 & $0.043^{*}$ \\
\hline LVMI & -0.264 & $0.001^{*}$ & 0.327 & $0.001^{*}$ & 0.283 & $0.001^{*}$ & 0.143 & 0.082 \\
\hline Retinopathy Grade & -0.137 & 0.130 & 0.120 & 0.193 & 0.079 & 0.387 & -0.021 & 0.815 \\
\hline 24-h SBP & -0.186 & $0.019^{*}$ & 0.300 & $0.001^{*}$ & - & - & 0.769 & $0.001^{*}$ \\
\hline 24-h DBP & -0.057 & 0.475 & 0.146 & 0.070 & 0.769 & $0.001^{*}$ & - & - \\
\hline sTWEAK & - & - & -0.354 & $0.001^{*}$ & -0.186 & $0.019^{*}$ & -0.057 & 0.475 \\
\hline IL-17A & -0.354 & $0.001^{*}$ & - & - & 0.300 & $0.001^{*}$ & 0.146 & 0.070 \\
\hline Duration of HT & -0.159 & $0.045^{*}$ & 0.117 & 0.144 & 0.050 & 0.532 & -0.121 & 0.130 \\
\hline Age & -0.183 & $0.021^{*}$ & 0.184 & $0.020^{*}$ & -0.027 & 0.733 & -0.252 & $0.001^{*}$ \\
\hline
\end{tabular}

*Denotes a significant correlation $(\mathrm{P}<0.05)$.

Patient age, duration of HT, and mean sTWEAK, IL17A, 24-h SBP, and 24-h DBP were associated with AOD and were analyzed via univariate logistic regression, and the significant risk factors were included in the multivariate logistic regression model. Multivariate logistic regression analysis showed that patient age $(\mathrm{OR}=$ $1.045, \mathrm{P}=0.012)$, the sTWEAK level $(\mathrm{OR}=0.985, \mathrm{P}=$ $0.002)$, and mean 24-h SBP $(\mathrm{OR}=1.065, \mathrm{P}=0.001)$ were predictive of AOD (Table 3). A serum sTWEAK level $\leq$ $877.78 \mathrm{pg} / \mathrm{mL}$ predicted AOD with sensitivity of $58.23 \%$ and specificity of $78.75 \%$ (AUC $=0.706 \pm 0.041(\mathrm{SE}) ; 95 \%$ CI: $0.629-0.775$; $\mathrm{P}<0.001$ ) (Figure 3 ). Age $>59$ years predicted AOD with sensitivity of $45.24 \%$ and specificity of 76.25\% (AUC $=0.590 \pm 0.045(\mathrm{SE}) ; 95 \% \mathrm{CI}: 0.519-0.670$; $\mathrm{P}=0.027$ ). A mean 24-h SBP level $>127 \mathrm{mmHg}$ predicted AOD with sensitivity of $49.38 \%$ and specificity of 73.75\% (AUC $=0.603 \pm 0.042(\mathrm{SE}) ; 95 \% \mathrm{CI}$ : 0.524-0.682; $\mathrm{P}=0.003)$. The AUC value for the sTWEAK level predictive of AOD was higher than that for age and 24-h SBP (24-h SBP vs. sTWEAK: AUC: 0.103, P = 0.033; SBP vs. age: AUC: $0.013, P=0.806$; sTWEAK vs. age: AUC: $0.116, \mathrm{P}=0.028$ ). The present findings indicate that the

Table 3 Logistic regression analysis of the risk factors associated with AOD

\begin{tabular}{|c|c|c|c|c|}
\hline \multirow[t]{2}{*}{ Variables } & \multicolumn{2}{|l|}{ Univariate } & \multicolumn{2}{|l|}{ Multivariate } \\
\hline & OR $(95 \% \mathrm{Cl})$ & $P$ & OR $(95 \% \mathrm{Cl})$ & $P$ \\
\hline Age & $1.040(1.011-1.070)$ & $0.006^{*}$ & $1.045(1.010-1.076)$ & $0.012^{*}$ \\
\hline Duration of HT & $1.062(1.046-1.143)$ & $0.042^{*}$ & $0.995(0.911-1.087)$ & 0.236 \\
\hline sTWEAK & $0.990(0.985-0.994)$ & $0.001^{*}$ & $0.985(0.983-0.996)$ & $0.002^{*}$ \\
\hline IL-17A & $1.138(1.030-1.393)$ & $0.021^{*}$ & $0.996(0.805-1.232)$ & 0.495 \\
\hline 24-h SBP & $1.061(1.032-1.091)$ & $0.001^{*}$ & 1.065 (1.030-1.093) & $0.001^{*}$ \\
\hline 24-h DBP & $1.038(1.020-1.075)$ & $0.034^{*}$ & $0.943(0.875-1.017)$ & 0.125 \\
\hline
\end{tabular}

serum sTWEAK level was a better diagnostic predictor of AOD than patient age and mean 24-h SBP.

\section{Discussion}

In the present study the serum sTWEAK level was significantly lower and the IL-17A level was significantly higher in the HT patients with AOD than in those without AOD. To the best of our knowledge the present study is the first to investigate the relationship between AOD in HT patients, and the serum sTWEAK and IL-17A levels.

Recent studies indicate that HT and AOD associated with HT not only occur due to hemodynamic disturbances, but that the natural and adaptive immune systems play a role [19]. Several rat studies reported that following infusion of deoxycorticosterone acetate and angiotensin II, a number of cytokines (TNF- $a$, IL-1B, IL6, IL-10, IL-17, and TGF-B1) were synthesized by the natural and adaptive immune systems, resulting in vascular inflammation $[5,20]$. Although many cytokines are known to play a role in the etiopathogenesis of HT, the relationship between HT, and IL-17A and sTWEAK remains unclear.

TWEAK is a cytokine of the TNF family that activates many components of cellular activity, including cell reproduction and cell death [20]. Some studies that investigated the STWEAK level reported that it was low in patients with acute coronary syndrome [21], heart failure [22], coronary artery disease [23], chronic renal disease [24], and kidney transplantation [25]. Various explanations for low sTWEAK levels in such patients have been posited, including that in cases of chronic inflammation the Fn14 level increases and binds to sTWEAK, resulting in a decrease in the serum STWEAK level [26], and that an increase in the scavenger receptor CD 163 level causes inflammatory macrophages to break down sTWEAK in the presence of inflammation [27]. 


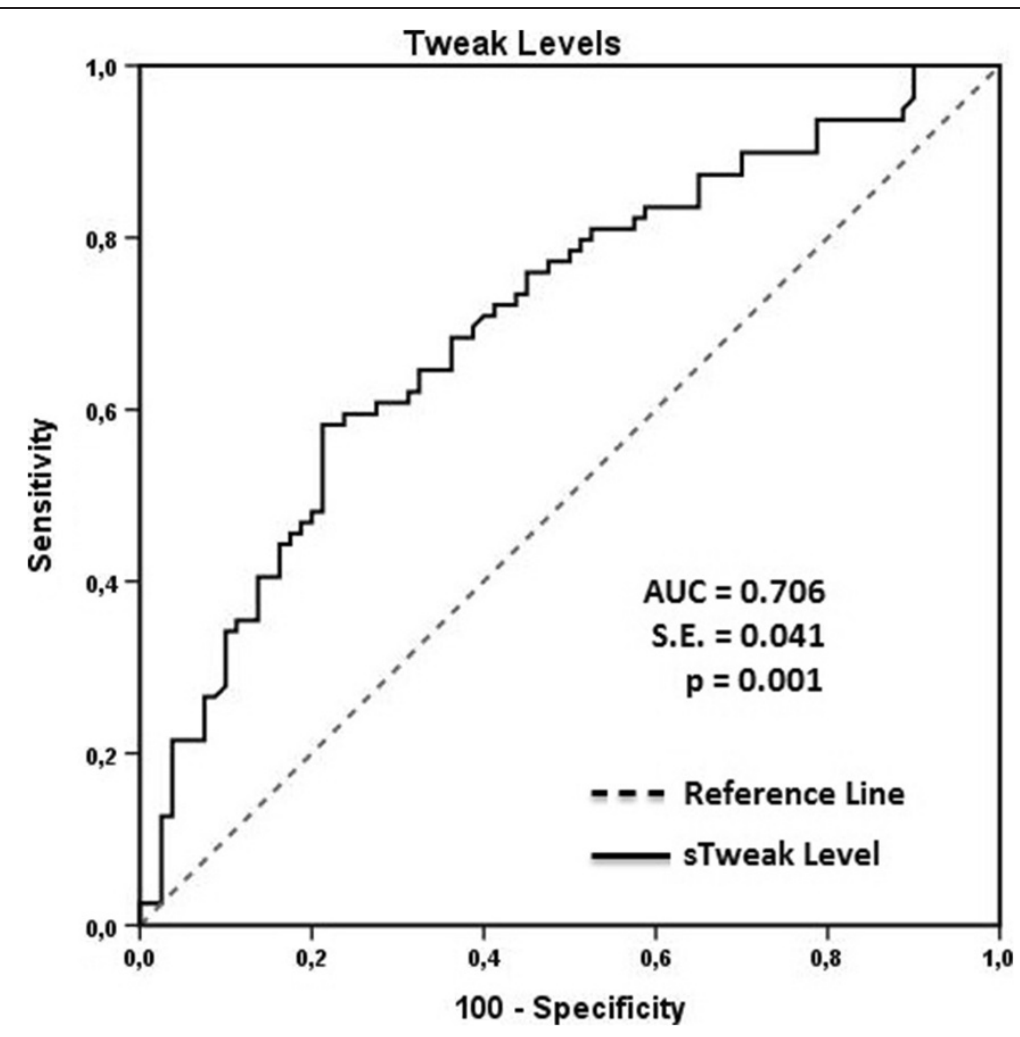

Figure 3 ROC curve analysis of the prediction of AOD based on the serum STWEAK level.

A search of the literature indicated that Karadurmus et al.'s [7] study is the only one to have examined the relationship between the serum STWEAK level and HT. Their study included 51 patients that were recently diagnosed as HT and treatment naïve; the mean sTWEAK level in the HT patients was lower than that in the healthy control group, but the relationship between the sTWEAK level and AOD was not investigated. In the present study the serum STWEAK level was observed to be lower in the HT patients AOD than in those without AOD. Sustained high blood pressure can lead to chronic inflammation, resulting in target organ damage, such as atherosclerosis and proteinuria. The lower STWEAK level in the present study's HT patients with AOD might have been due to chronic inflammation that already existed.

An earlier study reported that high levels of Fn14 in patients with cardiac hypertrophy was associated with elevated blood pressure and that the STWEAK level was lower in the cardiac hypertrophy patients because of binding to Fn14 receptor [28]. Another study reported elevated Fn14 levels in patients with non-immune primary glomerular disease and proteinuria [29]. In the present study there was a negative correlation between the serum sTWEAK level, and microalbuminuria level, proteinuria level, LVMI, and mean 24-h SBP, which might have been due to high level of Fn14 that bound to
sTWEAK and decrease in the serum sTWEAK level following chronic inflammation.

IL-17A is a cytokine that is suggested to play a role in the pathophysiology of HT, and its relationship to HT has been the subject of recent research. Several recent studies have reported that the IL-17A level increases in cases of atherosclerosis and vascular inflammation, and that such increases contribute to progression of inflammation $[14,30]$.

Nguyen et al. reported that SBP increased significantly in mice that received IL-17A (IP injection of $1 \mu \mathrm{g} / \mathrm{d}$ for $7 \mathrm{~d}$ ), which was attributed to a decrease in the response to aortic nitric oxide (NO)-dependent relaxation and an increase in NO synthase Thr495 phosphorylation (which leads to a decrease in NO production and vasodilation, and is associated with HT) [5].

Madhur et al. [31] conducted a prospective study that included 112 HT patients, and reported that the IL-17 level was significantly higher in the HT patients than in the normotensive group. They suggested that the finding was due to an increase in the level of Th17 in T cells and an increase in the IL-17 level following infusion of chronic angiotensin II, an increase in superoxide radicals due to deposition of IL-17 on vessel walls, a decrease in endothelium-related vasodilatation, and some affected genes (e.g. stanniocalcin-1), which caused HT and vascular inflammation. In addition, they also reported that IL- 
17 cell infiltration in the aorta occured in cases of angiotensin II-induced HT and that HT was not maintained in IL-17 knockout mice.

Liu et al. [32] observed a positive correlation between the IL-17A level and CIMT in atherosclerotic patients. In the present study the serum IL-17A level was significantly higher in HT patients with AOD than in those without AOD. In addition, there was a positive correlation between the serum IL-17A level and the microalbuminuria level, proteinuria level, CIMT, LVMI, and mean 24-h SBP, all of which are indicators of AOD. A decrease in NO related to IL-17A may lead to endothelial dysfunction that can result in atherosclerosis and an increase in CIMT, as reported by Madhur and Nguyen.

A study that investigated the STWEAK level as a predictor of target organ damage in HT patients reported that a low sTWEAK level was an independent risk factor for cardiovascular events [33]; however, findings concerning the predictive value of the IL-17A level are inconsistent.

The literature includes studies that have investigated the relationship between a variety of biomarkers and AOD in patients with HT [34-36]. Morillas et al. [34] reported that risk factors in HT patients, including age, smoking, diabetes mellitus, waist circumference, IL-6, and sTNF-R1 (marker of inflammation and apoptosis), are independent predictors of target organ damage based on multivariate logistic regression analysis. Ratto et al. [35] reported that the hs-CRP level was an independent predictor of AOD, based on multivariate logistic regression analysis, and Çalışkan et al. [36] observed that the serum uric acid level was a good predictor of coronary flow reserve based on ROC curve analysis (AUC $=0.760$, $\mathrm{P}<0.0001$ ). In the present study the sTWEAK level was observed to be a better predictor of AOD than the other predictors (e.g. age and SBP) $(\mathrm{AUC}=0.706, \mathrm{P}<0.001$ ).

The present findings show that patient age, sTWEAK, and mean 24-h SBP were independent predictors of AOD, based on a multivariate model of risk factors (age, sTWEAK level, IL-17A level, mean 24-h SBP and 24-h DBP, and duration of HT) that independently play a role in target organ damage. Mean age of the present study's HT patients with AOD was significantly higher than of those without AOD, and there was a weak negative correlation between the serum STWEAK level and patient age; however, regression analysis with inclusion of age showed that the relationship between the serum sTWEAK level and AOD was independent of age. To the best of our knowledge the present study is the first to report on the relationship between the serum sTWEAK level and age. The only limitation of the present study is its cross-sectional design, which precludes any conclusion regarding the causal relationship between the serum sTWEAK and IL17-A levels, and AOD in HT patients.

\section{Conclusion}

As AOD is an interim stage in the progression of vascular diseases and is a pathology with an important role in identifying cardiovascular risk in HT patients, AOD in HT patients must be carefully examined. Additional prospective observational studies are needed to more clearly delineate the role of STWEAK and IL-17A in the development of target organ damage.

\section{Abbreviations}

AOD: Asymptomatic organ damage; CCB: Calcium channel blocker; RAS: Renin angiotensin system; CMIT: Carotid intimal media thickness; Fn14: Fibroblast growth factor-inducible molecule 14; GFR: Glomerular filtration rate; HDL: High-density lipoprotein; HT: Hypertension; IL-17A: Interleukin-17A; LVMI: Left ventricular mass index; LDL: Low-density lipoprotein; NO: Nitric oxide; sTWEAK: Soluble tumor necrosis factor-like weak inducer of apoptosis; TG: Triglycerides; 24-h SBP: 24-h systolic blood pressure;

24-h DPB: 24-h diastolic blood pressure.

\section{Competing interests}

The authors declare that they have no competing interests.

\section{Authors' contributions}

IA and NO: Conception, design, and manuscript writing. All authors contributed to data collection and data interpretation, and approved the manuscript submitted to BMC Nephrology.

\section{Author details}

'Department of Nephrology, Ankara Numune Education and Research Hospital, Sihhiye, Ankara 061100, Turkey. ${ }^{2}$ Biochemistry Department, Ankara Numune Education and Research Hospital, Sihhiye, Ankara 061100, Turkey.

${ }^{3}$ Cardiology Department, Ankara Numune Education and Research Hospital, Sihhiye, Ankara 061100, Turkey. ${ }^{4}$ Radiology Department, Ankara Numune Education and Research Hospital, Sihhiye, Ankara 061100, Turkey.

Received: 7 July 2014 Accepted: 29 September 2014 Published: 1 October 2014

\section{References}

1. Mancia G, Fagard R, Narkiewicz K, Redon J, Zanchetti A, Bohm M, Christiaens T, Cifkova R, De Backer G, Dominiczak A, Galderisi M, Grobbee DE, Jaarsma T, Kirchhof P, Kjeldsen SE, Laurent S, Manolis AJ, Nilsson PM, Ruilope LM, Schmieder RE, Sirnes PA, Sleight P, Viigimaa M, Waeber B, Zannad F, Burnier M, Ambrosioni E, Caufield M, Coca A, Olsen MH: 2013 $\mathrm{ESH} / \mathrm{ESC}$ guidelines for the management of arterial hypertension: the Task Force for the Management of Arterial Hypertension of the European Society of Hypertension (ESH) and of the European Society of Cardiology (ESC). Eur Heart J 2013, 34(28):2159-2219.

2. Beevers G, Lip GY, O'Brien E: ABC of hypertension: the pathophysiology of hypertension. BMJ 2001, 322(7291):912-916.

3. Bautista LE: Inflammation, endothelial dysfunction, and the risk of high blood pressure: epidemiologic and biological evidence. $J$ Hum Hypertens 2003, 17(4):223-230.

4. Schiffrin EL: Immune mechanisms in hypertension and vascular injury. Clin Sci (Lond) 2014, 126(4):267-274.

5. Nguyen $\mathrm{H}$, Chiasson VL, Chatterjee P, Kopriva SE, Young KJ, Mitchell BM: Interleukin-17 causes Rho-kinase-mediated endothelial dysfunction and hypertension. Cardiovasc Res 2013, 97(4):696-704.

6. Schiffrin EL: T lymphocytes: a role in hypertension? Curr Opin Nephrol Hypertens 2010, 19(2):181-186.

7. Karadurmus N, Tapan S, Cakar M, Naharci I, Celik T, Tasci I, Sayin S, Dogan T, Turker T, Erbil MK, Saglam K: Lower plasma soluble TWEAK concentration in patients with newly diagnosed hypertension. Clin Invest Med 2012, 35(1):E20-E26.

8. Chicheportiche Y, Bourdon PR, Xu H, Hsu YM, Scott H, Hession C, Garcia I, Browning JL: TWEAK, a new secreted ligand in the tumor necrosis factor family that weakly induces apoptosis. J Biol Chem 1997, 272(51):32401-32410. 
9. Brown SA, Ghosh A, Winkles JA: Full-length, membrane-anchored TWEAK can function as a juxtacrine signaling molecule and activate the NF-kappaB pathway. J Biol Chem 2010, 285(23):17432-17441.

10. Ren MY, Sui SJ: The role of TWEAK/Fn14 in cardiac remodeling. Mol Biol Rep 2012, 39(11):9971-9977.

11. Harrington LE, Hatton RD, Mangan PR, Turner H, Murphy TL, Murphy KM Weaver CT: Interleukin 17-producing CD4+ effector T cells develop via a lineage distinct from the Thelper type 1 and 2 lineages. Nat Immunol 2005, 6(11):1123-1132.

12. Chabaud M, Fossiez F, Taupin JL, Miossec P: Enhancing effect of IL-17 on IL-1-induced IL- 6 and leukemia inhibitory factor production by rheumatoid arthritis synoviocytes and its regulation by Th2 cytokines. J Immunol 1998, 161(1):409-414.

13. Tesmer LA, Lundy SK, Sarkar S, Fox DA: Th17 cells in human disease. Immunol Rev 2008, 223:87-113.

14. Von Vietinghoff S, Ley K: Interleukin 17 in vascular inflammation. Cytokine Growth Factor Rev 2010, 21(6):463-469.

15. Friedewald WT, Levy RI, Fredrickson DS: Estimation of the concentration of low-density lipoprotein cholesterol in plasma, without use of the preparative ultracentrifuge. Clin Chem 1972, 18(6):499-502.

16. Levey AS, Berg RL, Gassman JJ, Hall PM, Walker WG: Creatinine filtration, secretion and excretion during progressive renal disease. Modification of Diet in Renal Disease (MDRD) Study Group. Kidney Int Supp/ 1989, 27:S73-S80.

17. Devereux RB, Reichek N: Echocardiographic determination of left ventricular mass in man. Anatomic validation of the method. Circulation 1977, 55(4):613-618

18. Pignoli P, Tremoli E, Poli A, Oreste P, Paoletti R: Intimal plus medial thickness of the arterial wall: a direct measurement with ultrasound imaging. Circulation 1986, 74(6):1399-1406.

19. Krebs CF, Lange S, Niemann G, Rosendahl A, Lehners A, Meyer-Schwesinger C, Stahl RA, Benndorf RA, Velden J, Paust HJ, Panzer U, Ehmke H, Wenzel UO: Deficiency of the interleukin $17 / 23$ axis accelerates renal injury in mice with deoxycorticosterone acetate + angiotensin ii-induced hypertension. Hypertension 2014, 63(3):565-571.

20. Burkly LC, Michaelson JS, Zheng TS: TWEAK/Fn14 pathway: an immunological switch for shaping tissue responses. Immunol Rev 2011, 244(1):99-114

21. Chorianopoulos E, Jarr K, Steen H, Giannitsis E, Frey N, Katus HA: Soluble TWEAK is markedly upregulated in patients with ST-elevation myocardial infarction and related to an adverse short-term outcome. Atherosclerosis 2010, 211(1):322-326.

22. Chorianopoulos E, Rosenberg M, Zugck C, Wolf J, Katus HA, Frey N: Decreased soluble TWEAK levels predict an adverse prognosis in patients with chronic stable heart failure. Eur J Heart Fail 2009, 11(11):1050-1056.

23. Jelic-Ivanovic Z, Bujisic N, Spasic S, Bogavac-Stanojevic N, SpasojevicKalimanovska V, Kotur-Stevuljevic J: Circulating sTWEAK improves the prediction of coronary artery disease. Clin Biochem 2009, 42(13-14):1381-1386.

24. Yilmaz MI, Carrero JJ, Ortiz A, Martin-Ventura JL, Sonmez A, Saglam M, Yaman H, Yenicesu M, Egido J, Blanco-Colio LM: Soluble TWEAK plasma levels as a novel biomarker of endothelial function in patients with chronic kidney disease. Clin J Am Soc Nephrol 2009, 4(11):1716-1723.

25. Turkmen K, Tonbul HZ, Erdur FM, Toker A, Biyik Z, Ozbiner H, Gaipov A, Gul EE, Kayrak M, Solak Y, Ozbek O, Turk S, Covic A: Soluble TWEAK independently predicts atherosclerosis in renal transplant patients. BMC Nephrol 2013, 14:144.

26. Vendrell J, Chacon MR: TWEAK: a new player in obesity and diabetes. Front Immunol 2013, 4:488.

27. Moreno JA, Munoz-Garcia B, Martin-Ventura JL, Madrigal-Matute J, Orbe J, Paramo JA, Ortega L, Egido J, Blanco-Colio LM: The CD163-expressing macrophages recognize and internalize TWEAK: potential consequences in atherosclerosis. Atherosclerosis 2009, 207(1):103-110.

28. Novoyatleva T, Sajjad A, Engel FB: TWEAK-Fn14 cytokine-receptor axis: a new player of myocardial remodeling and cardiac failure. Front Immunol 2014, 5:50.

29. Sanchez-Nino MD, Poveda J, Sanz AB, Mezzano S, Carrasco S, FernandezFernandez B, Burkly LC, Nair V, Kretzler M, Hodgin JB, Nair V, Kretzler M, Hodgin JB, Ruiz-Ortega M, Selgas R, Egido J, Ortiz A: Fn14 in podocytes and proteinuric kidney disease. Biochim Biophys Acta 2013, 1832:2232-2243.
30. Liuzzo G, Trotta F, Pedicino D: Interleukin-17 in atherosclerosis and cardiovascular disease: the good, the bad, and the unknown. Eur Heart $J$ 2013, 34(8):556-559.

31. Madhur MS, Lob HE, McCann LA, Iwakura Y, Blinder Y, Guzik TJ, Harrison DG Interleukin 17 promotes angiotensin II-induced hypertension and vascular dysfunction. Hypertension 2010, 55(2):500-507.

32. Liu Z, Lu F, Pan H, Zhao Y, Wang S, Sun S, Li J, Hu X, Wang L: Correlation of peripheral Th17 cells and Th17-associated cytokines to the severity of carotid artery plaque and its clinical implication. Atherosclerosis 2012, 221(1):232-241

33. Przybylowski P, Malyszko JS, Malyszko J: Soluble tumor necrosis factor-like weak inducer of apoptosis plasma levels as a novel biomarker of endothelial function in prevalent orthotopic heart transplant recipients. Transplant Proc 2011, 43(5):1900-1903.

34. Morillas P, de Andrade H, Castillo J, Quiles J, Bertomeu-Gonzalez V, Cordero A, Tarazon E, Rosello E, Portoles M, Rivera M, Bertomeu-Martinez V: Inflammation and apoptosis in hypertension. Relevance of the extent of target organ damage. Rev Esp Cardio/ 2012, 65(9):819-825.

35. Ratto E, Leoncini G, Viazzi F, Falqui V, Parodi A, Conti N, Tomolillo C, Deferrari G, Pontremoli R: C-reactive protein and target organ damage in untreated patients with primary hypertension. J Am Soc Hypertens 2007, 1(6):407-413.

36. Caliskan M, Erdogan D, Gullu H, Yildirim I, Ozer I, Yildirir A, Muderrisoglu H: Association between serum uric acid levels and coronary flow reserve in hypertensive patients without concomitant risk factors. Blood Press 2007, 16(4):254-261.

doi:10.1186/1471-2369-15-159

Cite this article as: Ates et al:: The relationship between asymptomatic organ damage, and serum soluble tumor necrosis factor-like weak inducer of apoptosis (sTWEAK) and Interleukin-17A (IL-17A) levels in non-diabetic hypertensive patients. BMC Nephrology 2014 15:159.

\section{Submit your next manuscript to BioMed Central and take full advantage of:}

- Convenient online submission

- Thorough peer review

- No space constraints or color figure charges

- Immediate publication on acceptance

- Inclusion in PubMed, CAS, Scopus and Google Scholar

- Research which is freely available for redistribution 